\title{
Local Interneurons Regulate Synaptic Strength by Retrograde Release of Endocannabinoids
}

\author{
Michael Beierlein and Wade G. Regehr \\ Department of Neurobiology, Harvard Medical School, Boston, Massachusetts 02115
}

\begin{abstract}
Neurons release endocannabinoids from their dendrites to trigger changes in the probability of transmitter release. Although such retrograde signaling has been described for principal neurons, such as hippocampal pyramidal cells and cerebellar Purkinje cells (PCs), it has not been demonstrated for local interneurons. Here we tested whether inhibitory interneurons in the cerebellum, stellate cells (SCs) and basket cells, regulate the strength of parallel fiber (PF) synapses by releasing endocannabinoids. We found that depolarizationinduced suppression of excitation (DSE) is present in both SCs and basket cells. The properties of retrograde inhibition were examined more thoroughly for SCs. Both DSE and synaptically evoked suppression of excitation (SSE) triggered with brief PF bursts require elevations of postsynaptic calcium, are blocked by a type 1 cannabinoid receptor (CB1R) antagonist, and are absent in mice lacking the CB1R. SSE for SCs is similar to that described previously for PCs in that it is prevented by BAPTA and DAG lipase inhibitors in the recording pipette; however, unlike in PCs, NMDA receptors (NMDARs) play an important role in SSE for SCs. Although SCs express CB1Rs postsynaptically, neither high-frequency firing of SCs nor PF bursts lead to autocrine suppression of subsequent SC activity. Instead, PF bursts decrease the amplitude of disynaptic inhibition in PCs by evoking endocannabinoid release that transiently reduces the ability of $\mathrm{PF}$ synapses to trigger spikes in SCs. Thus, local interneurons within the cerebellum can release endocannabinoids through metabotropic glutamate receptor- and NMDAR-dependent mechanisms and contribute to use-dependent modulation of circuit properties.
\end{abstract}

Key words: 2-AG; CB1; cerebellum; DSE; NMDA receptor; stellate cell

\section{Introduction}

Endocannabinoids are retrograde messengers that allow neurons to dynamically regulate synaptic strength (Kreitzer and Regehr, 2002; Diana and Marty, 2004; Chevaleyre et al., 2006). Depolarization of postsynaptic cells leads to large calcium increases that evoke endocannabinoid release and suppression of inhibitory or excitatory synapses (Kreitzer and Regehr, 2001a,b; Maejima et al., 2001; Ohno-Shosaku et al., 2001; Wilson and Nicoll, 2001). In addition, synaptic activation with brief bursts can evoke endocannabinoid release and lead to synaptically evoked suppression of excitation (SSE) (Brown et al., 2003; Melis et al., 2004; Brenowitz and Regehr, 2005). SSE usually requires both activation of postsynaptic metabotropic receptors and modest dendritic calcium increases (Brown et al., 2003; Brenowitz and Regehr, 2005; Hashimotodani et al., 2005; Maejima et al., 2005). For SSE and depolarization-induced suppression of excitation (DSE), endocannabinoids released from the postsynaptic cell act retrogradely to reduce the probability of neurotransmitter release by activating type 1 endocannabinoid receptors (CB1Rs) expressed on presynaptic boutons.

Received March 3, 2006; revised July 17, 2006; accepted Aug. 22, 2006.

This work was supported by National Institutes of Health Grant R01-NS044396 (W.G.R.). We thank Claudio Acuna-Goycolea, Aaron Best, Stephan Brenowitz, Megan Carey, John Crowley, Kendall Jensen, and Patrick Safo for comments on this manuscript.

Correspondence should be addressed to Dr. Wade G. Regehr, Goldenson 307, Department of Neurobiology, Harvard Medical School, 220 Longwood Avenue, Boston, MA 02115. E-mail: wade regehr@hms.harvard.edu. DOI:10.1523/JNEUROSCI.0958-06.2006

Copyright $\odot 2006$ Society for Neuroscience $\quad$ 0270-6474/06/269935-09\$15.00/0
The observation that many types of neurons express CB1Rs on their presynaptic terminals suggests that retrograde signaling plays an important role throughout the brain (Egertova and Elphick, 2000; Chevaleyre et al., 2006). Indeed, CA1 pyramidal cells, cerebellar Purkinje cells (PCs), and numerous other principal cells all release endocannabinoids that lead to short- and long-term changes in synaptic strength (Chevaleyre et al., 2006); however, because CB1Rs are activated by several types of endocannabinoids that are synthesized by diverse pathways (Piomelli, 2003; Bisogno et al., 2005), it is unclear which cell types can release endocannabinoids. Studies in hippocampal interneurons have failed to demonstrate retrograde signaling by endocannabinoids (Hoffman et al., 2003; Patenaude et al., 2005), whereas one type of neocortical interneuron has been shown to synthesize endocannabinoids to regulate its own firing in an autocrine manner (Bacci et al., 2004). Thus, little is known about the role of retrograde signaling in different types of interneurons.

Here we examine retrograde signaling from local interneurons in the cerebellum and the regulation of parallel fiber (PF) synapses formed by granule cells. We focus on baskets cells (BCs) and stellate cells (SCs) that are located within the molecular layer of the cerebellar cortex and provide inhibitory input predominantly to PCs (Palay and Chan-Palay, 1974). We find that these interneurons release endocannabinoids and regulate PF synapses in response to either postsynaptic depolarization or synaptic activation. The properties of DSE and SSE are similar in many ways to those observed at synapses onto other cell types, including the PF to PC synapse; however, SSE at PF to SC synapses differs in 
that NMDA receptors play an important role in endocannabinoid release. One functional consequence of SSE is that activation of PF synapses with a burst transiently reduces the ability of PF synapses to trigger spikes in SCs, thus reducing the magnitude of feedforward inhibition of their postsynaptic targets.

\section{Materials and Methods}

Tissue preparation. Sprague Dawley rats [postnatal day 14 (P14) to P18] were anesthetized with halothane and decapitated, and transverse cerebellar slices $(250 \mu \mathrm{m})$ were obtained. For some experiments, slices were obtained from CB1 receptor-deficient C57BL/6J mice (Zimmer et al., 1999 ) or wild-type mice (P15-P18). Slices were cut in a sucrosecontaining solution consisting of (in mM) $81.2 \mathrm{NaCl}, 23.4 \mathrm{NaHCO}_{3}, 69.9$ sucrose, 23.3 glucose, $2.4 \mathrm{KCl}, 1.4 \mathrm{NaH}_{2} \mathrm{PO}_{4}, 6.7 \mathrm{MgCl}_{2}$, and $0.5 \mathrm{CaCl}_{2}$. Slices were incubated at $32^{\circ} \mathrm{C}$ for $30 \mathrm{~min}$ and then transferred for $30 \mathrm{~min}$ at $32^{\circ} \mathrm{C}$ to a saline solution consisting of the following (in mM): $125 \mathrm{NaCl}$, $26 \mathrm{NaHCO}_{3}, 2.5 \mathrm{KCl}, 1.25 \mathrm{NaH}_{2} \mathrm{PO}_{4}, 25$ glucose, $2 \mathrm{CaCl}_{2}$, and $1 \mathrm{MgCl}_{2}$. For experiments examining changes in paired-pulse facilitation (PPF), calcium was increased to $4 \mathrm{~mm}$ to reduce baseline PPF. Experiments were performed at $33-34^{\circ} \mathrm{C}$ with an in-line heater (Warner, Hamden, CT); the bath was superfused with saline solution at 3-4 $\mathrm{ml} / \mathrm{min}$ by means of a Minipulse 3 pump (Gilson, Middleton, WI).

Electrophysiology. Whole-cell recordings with pipettes of 1-1.5 $\mathrm{M} \Omega$ (PCs) or 2-3 M $\Omega$ (SCs and BCs) were performed with differential interference contrast optics and a $60 \times$ water immersion lens. For DSE experiments, the internal solution contained the following (in mM): $127 \mathrm{Cs}-$ $\mathrm{MeSO}_{3}, 10 \mathrm{CsCl}, 10$ HEPES, 0.5 EGTA, $2 \mathrm{MgCl}_{2}, 0.16 \mathrm{CaCl}_{2}, 2 \mathrm{Mg}$-ATP, $0.4 \mathrm{Na}$-GTP, 14 Tris-creatine phosphate, and 22 (triethylamino)- $\mathrm{N}-(2,6-$ dimethyl acetamine (QX-314), adjusted to $310 \mathrm{mOsm}$. Where indicated, $20 \mathrm{~mm}$ BAPTA was added and replaced $\mathrm{CsMeSO}_{3}$. To examine disynaptic inhibition, PCs were voltage clamped at $0 \mathrm{mV}$, with an internal solution containing the following (in mM): $30 \mathrm{CsMeSO}_{3}, 35 \mathrm{CsCl}, 20 \mathrm{CsBAPTA}, 2$ HEDTA, 15 HEPES, 0.2 EGTA, $1 \mathrm{MgCl}_{2}, 4 \mathrm{CaCl}_{2}, 15$ TEA-Cl, $2 \mathrm{Mg}$-ATP, 0.3 Na-GTP, 10 Tris-creatine phosphate, and 2 QX-314, adjusted to 310 mOsm. For experiments in which endocannabinoid release was evoked synaptically, the internal solution contained the following (in $\mathrm{mM}$ ): 130 $\mathrm{KMeSO}_{3}, 10 \mathrm{NaCl}, 10 \mathrm{HEPES}, 0.5 \mathrm{EGTA}, 2 \mathrm{MgCl}_{2}, 0.16 \mathrm{CaCl}_{2}, 4$ $\mathrm{Na}_{2} \mathrm{ATP}, 0.4 \mathrm{NaGTP}$, and 14 Tris-creatine phosphate, adjusted to 310 mOsm. In some experiments, $20 \mathrm{~mm}$ BAPTA and $4 \mathrm{~mm}$ calcium were added to achieve a free calcium concentration of $\sim 60 \mathrm{nM}$. Free calcium was calculated with the MaxChelator program (C. Patton, Stanford University, Stanford, CA). Recordings were obtained with an Axopatch 200B or Multiclamp 700A amplifier (Molecular Devices, Union City, CA). Parallel fibers were stimulated with glass electrodes (2-3 M $\Omega$ ) filled with artificial CSF. All recordings except those described in Fig. $7 J-L$ were performed in the presence of picrotoxin $(20 \mu \mathrm{M})$ to block $\mathrm{GABA}_{\mathrm{A}^{-}}$ mediated synaptic transmission. DSE experiments in SCs and BCs were performed in the presence of $250 \mathrm{~nm}$ 2,3-dihydroxy-6-nitro-7sulfamoyl-benzo[f] quinoxaline (NBQX) to reduce EPSC amplitudes. For experiments in which endocannabinoid release was evoked synaptically (SSE), NBQX was omitted to maintain the physiological ratio of AMPA receptor to metabotropic glutamate receptor 1 (mGluR1). Furthermore, the $\mathrm{GABA}_{\mathrm{B}}$ receptor antagonist (2S)-3-\{[(15)-1(3,4-dichlorophenyl)ethyl] \}amino-2-hydroxypropyl)(phenylmethyl) phosphinic acid (CGP 55845) ( $2 \mu \mathrm{M})$ was added to prevent presynaptic inhibition associated with the activation of presynaptic $\mathrm{GABA}_{\mathrm{B}}$ receptors (Dittman and Regehr, 1997; Brown et al., 2003; Beierlein and Regehr, 2006). SSE evoked by 10 stimuli at $50 \mathrm{~Hz}$ was $0.34 \pm 0.07(n=9)$ in CGP 55845 compared with $0.32 \pm 0.08(n=5)$ in control, indicating that CGP 55845 did not affect the extent of SSE. DSE and SSE trials were typically repeated once every minute. In experiments testing the role of DAG lipase, slices were incubated in $100 \mu \mathrm{M}$ 1,6-bis(cyclohexyloximinocarbonylamino) hexane (RHC-80267) for $1 \mathrm{~h}$. Recordings were performed in the continued presence of $30 \mu \mathrm{M}$ RHC-80267.

$N$-piperidin-1-yl)-5-(4-iodophenyl)-1-(2,4-dichlorophenyl)-4-methyl-1 H-pyrazole-3-carboxamide (AM251), 7-hydroxyiminocyclopropan[b] chromen-1a-carboxylate ethyl ester (CPCCOEt), 2-carboxypiperazin-4yl-propyl-1-phosphonic acid (CPP), D-AP5, NBQX, picrotoxin, and
CGP 55845 were obtained from Tocris Cookson (Ellisville, MO); BAPTA and CsBAPTA were obtained from Invitrogen (Carlsbad, CA); tetrahydrolipstatin (THL; Orlistat) was provided by Roche Pharmaceuticals (Nutley, NJ); and RHC-80267 was obtained from Biomol (Plymouth Meeting, PA). All other chemicals were purchased from Sigma (St. Louis, $\mathrm{MO})$.

Data acquisition and analysis. Recordings were digitized at $20 \mathrm{kHz}$ with a 16-bit analog-to digital converter (ITC-16; Instrutech, Port Washington, NY). All analyses were performed with custom macros written in Igor Pro (Wavemetrics, Lake Oswego, OR). Averages are given as mean \pm SEM. Statistics were performed with the unpaired or paired Student's $t$ test. Differences were considered to be significant at $p<0.01$.

Fluorescent images of neurons. High-resolution images of PCs, SCs, and BCs were obtained with two-photon laser scanning microscopy (2PLSM) by using a modified Olympus confocal microscope equipped with a Tisapphire pulsed laser (Coherent, Santa Clara, CA). For these experiments, sagittal slices $(250 \mu \mathrm{m})$ were cut and neurons were recorded with a current-clamp internal solution, supplemented with $100 \mu \mathrm{M}$ Alexa-594 (Invitrogen, Carlsbad, CA). Data were collected at an excitation wavelength of $810 \mathrm{~nm}$ with Fluoview software (Olympus). A $3 \times 3$ median filter was applied to individual confocal images, and a $\mathrm{z}$-stack was generated with a maximal intensity projection of the confocal series.

\section{Results}

\section{DSE at PF synapses onto interneurons}

We examined the effect of postsynaptic depolarization on PF synapses onto PCs, SCs, and BCs. Recordings from SCs and BCs were obtained from the outer and inner third, respectively, of the molecular layer. Both BCs and SCs had extensive axonal arborizations, but only BCs formed multiple axon collaterals in the PC layer (Fig. 1D, G) (Palay and Chan-Palay, 1974). Cells were voltage clamped at $-70 \mathrm{mV}$ with a cesium-based internal solution, and PFs were stimulated at $0.5 \mathrm{~Hz}$ before and after depolarization of the postsynaptic cell to $0 \mathrm{mV}$ for $2 \mathrm{~s}$. As shown previously, depolarization of PCs led to DSE (Kreitzer and Regehr, 2001a) of PF synapses that was blocked by application of the CB1R antagonist AM251 $(2 \mu \mathrm{M})$ to the bath (Fig. $1 A-C)$. DSE was also reliably observed in both types of interneurons. On average, depolarization of SCs and BCs led to a reduction in synaptic strength to $0.34 \pm 0.03(n=11)$ and $0.30 \pm 0.10(n=7)$ of control, respectively, that was completely blocked by AM251. For each type of synapse, representative EPSCs before and after postsynaptic depolarization are shown for control conditions (Fig. $1 B, E, H$, left) and in the presence of AM251 (Fig. $1 B, E, H$, right), and the time course of DSE is summarized (Fig. $1 C, F, I$ ). These results establish that SCs and BCs can regulate PF synapses by releasing endocannabinoids. Similar to PCs, endocannabinoid release in SCs and BCs required an increase in postsynaptic calcium levels. When cells were recorded with an internal solution containing $20 \mathrm{~mm}$ BAPTA, EPSCs recorded $3 \mathrm{~s}$ after the beginning of a 2 s depolarization were $1.01 \pm 0.01$ for SCs $(n=5)$ and $0.98 \pm 0.02$ for BCs $(n=4)$, normalized to EPSCs evoked before depolarization, indicating that DSE was completely blocked.

Thus the ability to release endocannabinoids within the cerebellar cortex is not restricted to PCs. SCs and BCs are also capable of releasing endocannabinoids and transiently regulating their synaptic inputs.

\section{Properties of DSE at PF synapses onto interneurons}

Although the time course and the calcium dependence of DSE appeared to be similar at PF synapses onto PCs, SCs, and BCs, its magnitude was smaller for SCs and BCs than for PCs. To determine whether this was the consequence of a difference in the maximal extent of inhibition that could be achieved by CB1R activation at PF synapses onto the different postsynaptic targets, 


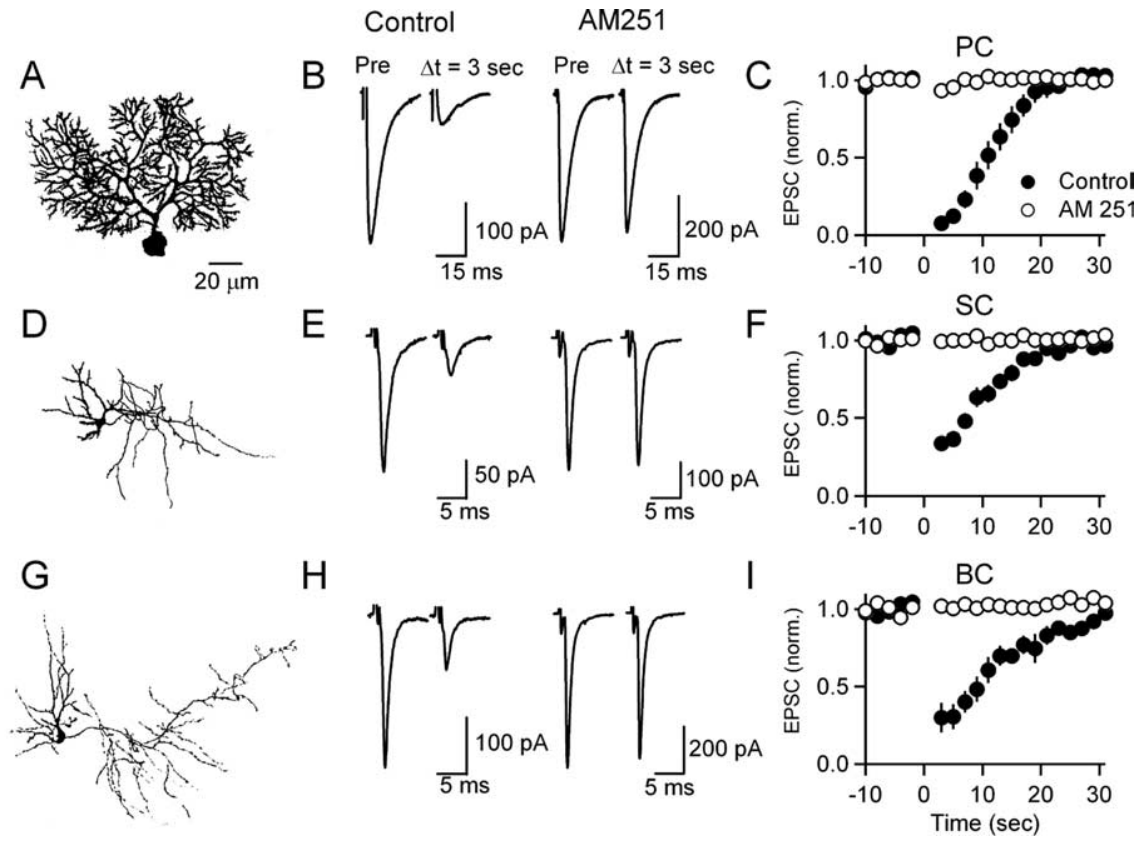

Figure 1. Depolarization of $S C S$ and $B C s$ suppresses parallel fiber synapses by activating cannabinoid receptors. $\boldsymbol{A}, \boldsymbol{D}, \boldsymbol{G}$, Fluorescent images of a $P C(\boldsymbol{A})$, an $S C(\boldsymbol{D})$, and a $B C(\boldsymbol{G})$, obtained with a two-photon laser scanning microscope, are shown. Images have been inverted. PFs were stimulated with an extracellular electrode at $0.5 \mathrm{~Hz}$, and EPSCs were recorded in voltage clamp at a holding potential of $-70 \mathrm{mV}$. At time $t=0$, cells were depolarized to $0 \mathrm{mV}$ for $2 \mathrm{~s}$. $\boldsymbol{B}, \boldsymbol{E}, \boldsymbol{H}$, Average responses of representative experiments are shown before and $3 \mathrm{~s}$ after the beginning of the postsynaptic depolarization for control conditions (left) and in the presence of the CB1R antagonist AM251 $(2 \mu \mathrm{M})$ (right) for PCs $(\boldsymbol{B}), \mathrm{SCS}(\boldsymbol{E})$, and BCS $(\boldsymbol{H}) . \boldsymbol{C}, \boldsymbol{F}, \boldsymbol{I}$, Summaries of the DSE time course are shown for control conditions (black symbols) and in the presence of $\mathrm{AM} 251$ (open symbols) for $\mathrm{PCs}(\boldsymbol{C}), \operatorname{SCs}(\boldsymbol{F})$, and BCs $(\boldsymbol{I})$. $\operatorname{Pre}$, Pre-pulse; norm., normalized.

we compared the magnitude of inhibition induced by bath application of the CB1R agonist $(R)-(+)$-[2,3-dihydro-5-methyl-3(4-morpholinylmethyl)pyrrolo[1,2,3-de)-1,4-benzoxazin-6-yl]1-napthalenylmethanone (WIN 55,212-2; $2 \mu \mathrm{M}$ ) for the three types of PF synapses. A typical experiment is shown in Figure $2 \mathrm{~A}$ for an SC. PF EPSC amplitudes were measured before (solid symbols) and after (open symbols) a 2 s depolarization, applied once per minute. DSE was prominent in control conditions. After bath application of WIN 55,212-2, synaptic strength was reduced to $\sim 12 \%$ of control, and DSE was occluded. Application of AM251 $(5 \mu \mathrm{M})$ reversed the effect of WIN 55,212-2 on the EPSC amplitude and blocked DSE. Importantly, WIN 55,212-2 equally inhibited PF synapses onto PCs, SCs, and BCs (Fig. 2 B). Thus the less pronounced DSE at PF synapses onto SCs and BCs compared with DSE at synapses onto PCs likely reflects differences in endocannabinoid release as opposed to differences in the expression of functional CB1Rs at these different synapses.

We also performed experiments to determine whether DSE at PF synapses onto BCs and SCs is mediated by a decrease in the probability of neurotransmitter release $(p)$. Previous studies of $\mathrm{PF}$ to PC synapses have shown that DSE arises from the release of endocannabinoids from PCs activating presynaptic CB1Rs that, in turn, reduce the probability of release by decreasing presynaptic calcium entry (Kreitzer and Regehr, 2001a; Brown et al., 2004). A reduction in $p$ is typically accompanied by an increase in paired-pulse ratio, as for DSE at the PF to PC synapse (Kreitzer and Regehr, 2001a). We therefore examined whether DSE at PF synapses onto interneurons is also accompanied by a change in paired-pulse plasticity. PF synapses were stimulated with pairs of stimuli $(20 \mathrm{~Hz})$ under control conditions and $3 \mathrm{~s}$ after the postsynaptic cell was depolarized to $0 \mathrm{mV}$ for $2 \mathrm{~s}$. Before depolar- ization, PF synapses onto all postsynaptic targets exhibited similar paired-pulse facilitation (Fig. 2D) [PC, $1.91 \pm 0.19(n=$ 7); SC, $1.81 \pm 0.1(n=10)$; BC, $1.89 \pm$ $0.13(n=4)]$. This suggests that PF synapses have approximately the same initial $p$ regardless of the postsynaptic target. After depolarization, paired-pulse facilitation at PF to SC synapses was increased, as shown for a representative experiment in which the responses before and after depolarization were scaled to the peak of the first EPSC (Fig. 2C). Across experiments, postsynaptic depolarization (DSE) and bath application of WIN 55,212-2 increased the paired-pulse ratio to $3.1 \pm 0.2$ and $3.1 \pm 0.3$, respectively, in PCs; to $2.6 \pm$ 0.2 and $3.1 \pm 0.2$ in SCs; and to $2.6 \pm 0.2$ and $2.9 \pm 0.2$ in BCs (Fig. 2 D). These data indicate that SCs and BCs can release endocannabinoids that activate presynaptic CB1Rs that, in turn, decrease the initial probability of release. Together, these findings suggest that DSE at PF to SC and $\mathrm{PF}$ to $\mathrm{BC}$ synapses is remarkably similar to DSE at PF to PC synapses.

\section{SSE at PF synapses onto SCs}

Granule cells fire bursts of action potentials in response to sensory stimulation (Chadderton et al., 2004). Activation of the PF to PC synapse with brief highfrequency trains can lead to endocannabinoid release and retrograde inhibition (Brown et al., 2003; Brenowitz and Regehr, 2005). It is not known whether realistic PF activity patterns also lead to endocannabinoid release and retrograde inhibition in cerebellar interneurons.

Here we tested whether synaptic activation leads to endocannabinoid release from SCs. Cells were recorded with a potassiumbased internal solution, and PF EPSCs evoked at $0.5 \mathrm{~Hz}$ were measured in voltage clamp at a holding potential of $-70 \mathrm{mV}$. The recording was then switched into current-clamp mode to allow the cell to depolarize and generate action potentials, and PF synapses were activated with a train of 10 stimuli at $50 \mathrm{~Hz}$. The recording was then switched back into voltage clamp, and $0.5 \mathrm{~Hz}$ test stimulation resumed. For these experiments, the stimulus intensity was adjusted to result in an average EPSC of 300-400 $\mathrm{pA}$, which corresponds to the synchronous activation of numerous PF inputs.

Brief synaptic bursts reliably led to retrograde inhibition at the PF to SC synapse (Fig. 3A). Bath application of AM251 led to an enhancement of synaptic strength after the burst (Fig. $3 B$ ), although the cell response evoked by the PF burst remained similar. This enhancement appeared to be presynaptic in nature and likely reflects a process similar to augmentation or post-tetanic potentiation (Zucker and Regehr, 2002; Brown et al., 2003).

A comparison of the properties of SSE in PCs and SCs revealed many similarities (Fig. 3C). Previous studies have found that SSE in PCs is dependent on both postsynaptic calcium elevations and mGluR1 activation, which lead to the activation of phospholipase $\mathrm{C}$, the production of DAG, and its conversion to 2 arachidonylglycerol (2-AG) by DAG lipase (Brenowitz and Regehr, 2005; Hashimotodani et al., 2005; Maejima et al., 2005). We found that, 

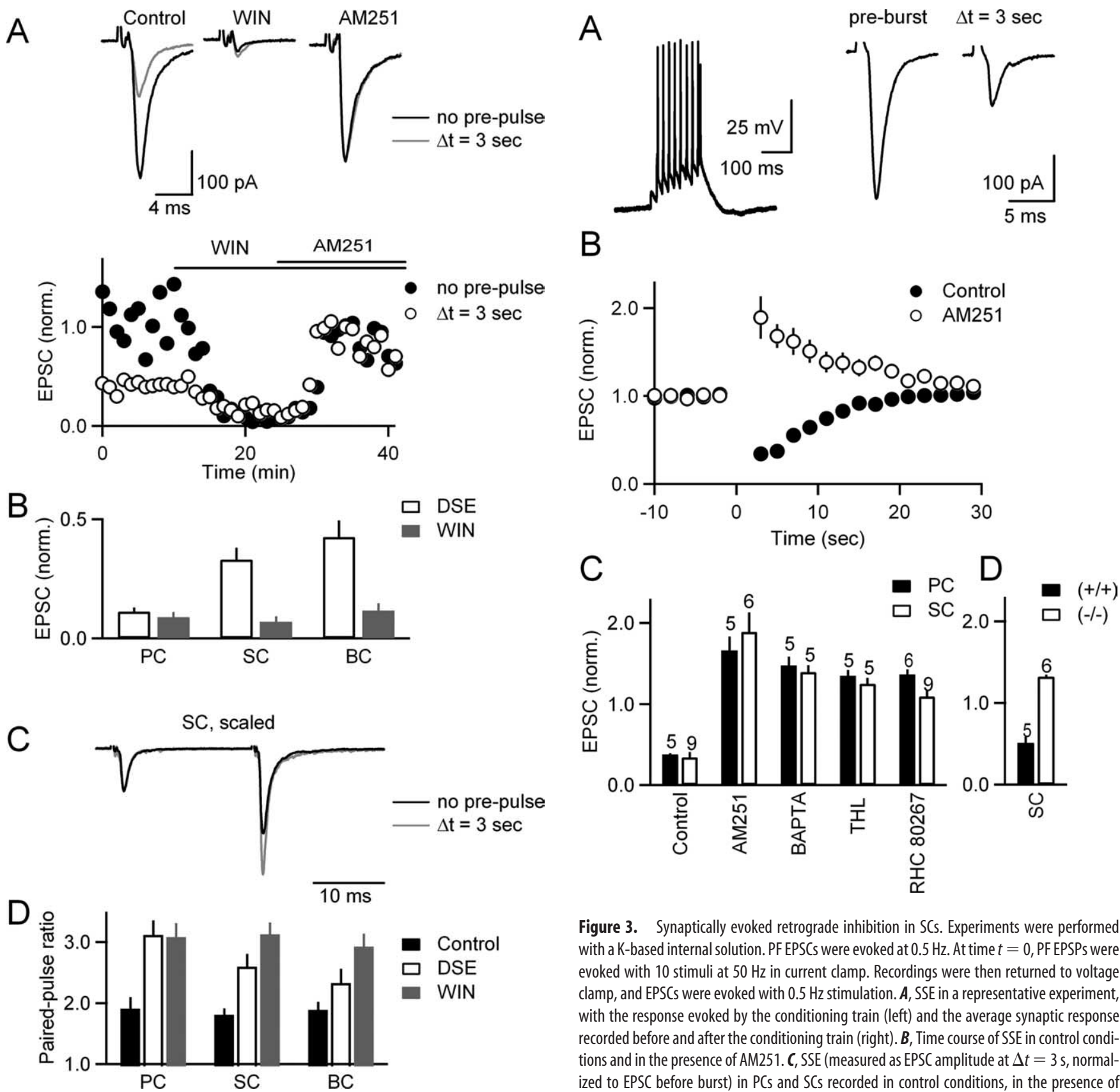

Figure 3. Synaptically evoked retrograde inhibition in SCS. Experiments were performed with a K-based internal solution. PF EPSCs were evoked at $0.5 \mathrm{~Hz}$. At time $t=0$, PF EPSPs were evoked with 10 stimuli at $50 \mathrm{~Hz}$ in current clamp. Recordings were then returned to voltage clamp, and EPSCs were evoked with $0.5 \mathrm{~Hz}$ stimulation. $A$, SSE in a representative experiment, with the response evoked by the conditioning train (left) and the average synaptic response recorded before and after the conditioning train (right). $B$, Time course of SSE in control conditions and in the presence of AM251. C, SSE (measured as EPSC amplitude at $\Delta t=3 \mathrm{~S}$, normalized to EPSC before burst) in PCS and SCs recorded in control conditions, in the presence of AM251 in the bath, in the presence of the calcium buffer BAPTA (20 mM) in the recording pipette, in the presence of the DAG lipase inhibitor THL $(2 \mu \mathrm{m})$ in the pipette, and in the presence of the DAG lipase inhibitor RHC $80267(30 \mu \mathrm{M})$ in the bath. The number of experiments is indicated. All treatments led to a significant reduction of SSE for both SCs and PCS $(p<0.01$; unpaired Student's $t$ test). $D$, SSE at the PF to SC synapse in CB1R knock-out mice was significantly reduced compared with SSE at synapses in wild-type littermates $(p<0.01$; unpaired Student's $t$ test). norm., Normalized. EPSC amplitude are shown in $\boldsymbol{A}$ and $\boldsymbol{B}$, and changes in paired-pulse ratio are shown in $\boldsymbol{C}$ and $\boldsymbol{D}$. $A$, A representative experiment is shown for PF to $\mathrm{SC}$ synapses; the average response before (black) and $3 \mathrm{~s}$ after (gray) depolarization is plotted for each condition (top), and the normalized EPSC amplitude is plotted as a function of time (bottom). $\boldsymbol{B}$, Experiments as in $\boldsymbol{A}$ are summarized to compare the effects of depolarizing the postsynaptic cell in control conditions with the effects of WIN 55,212-2 on the magnitude of the EPSC for the various types of PF synapses. C, Representative average responses are shown for PF to SC synapses before depolarization (black traces) and $3 \mathrm{~s}$ after depolarization (gray traces). Responses are normalized to the first EPSC to allow comparison of the extent of facilitation before and after depolarization (the amplitudes of EPSC $_{1}$ before and after depolarization were 350 and $120 \mathrm{pA}$, respectively). D, Summary of the change of facilitation induced by depolarization, with the average paired-pulse ratio $(P P R=$ $\mathrm{EPSC}_{2} / \mathrm{EPSC}_{1}$ ) in control conditions before depolarization (black bars), after depolarization in control conditions (white bars), and in the presence of WIN 55,212-2 (gray bars). WIN, WIN $55,212-2 ;$ norm., normalized.

under control conditions, pronounced SSE was observed for both PCs and SCs. For both cell types, bath application of AM251 not only eliminated retrograde inhibition but revealed strong enhancement. Moreover, inclusion of the calcium chelator BAPTA $(20 \mathrm{mM})$ in the recording pipette prevented the transient retrograde inhibition. This indicates that a calcium increase in both PCs and SCs is required for synaptically evoked retrograde inhibition. Inclusion of the DAG lipase inhibitor THL $(2 \mu \mathrm{M})$ in the recording pipette also prevented retrograde inhibition. Similarly, bath application of the DAG lipase inhibitor RHC-80267 

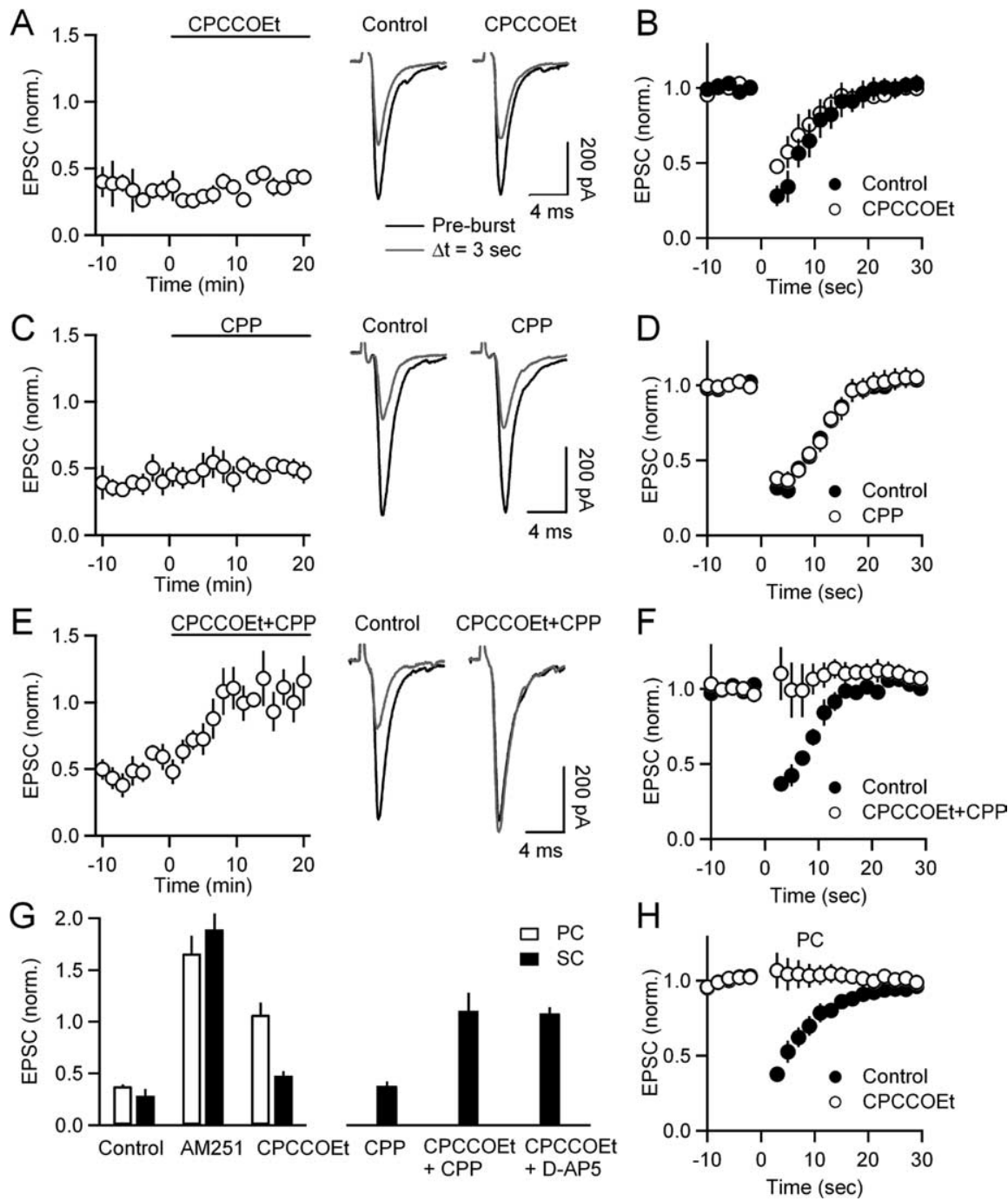

Figure 4. NMDARs mediate SSE in SCS. SSE was assessed after bath application of the mGluR1 antagonist CPCCOEt $(\boldsymbol{A}, \boldsymbol{B})$, the NMDAR antagonist $C P P(C, D)$, and coapplication of $C P C C O E t$ and $C P P(E, F) . A, C, E$, The extent of SSE for five to seven experiments in each condition is summarized during bath application of drugs (left); average synaptic currents from representative experiments are shown in control conditions and in the presence of antagonists (right). $\boldsymbol{B}, \boldsymbol{D}, \boldsymbol{F}$, Summary of the effects of CPCCOEt $(\boldsymbol{B})$, CPP $(\boldsymbol{D})$, and CPCCOEt plus CPP $(\boldsymbol{F})$ on the time course of SSE in SCS. G, Summary of the average SSE after conditioning trains (normalized to baseline) in PCs and SCs for control conditions, in the presence of $2 \mu \mathrm{MAM} 251,100 \mu \mathrm{M}$ CPCCOEt, $5 \mu \mathrm{M}$ CPP, and after coapplication of CPCCOEt and CPP and CPCCOEt and $50 \mu \mathrm{m}$ D-AP5. CPCCOEt application led to a statistically significant difference in SSE between SCS and PCS ( $p<0.01$; unpaired Student's $t$ test). SSE was significantly reduced in SCs after the coapplication of CPCCOEt and CPP and CPCCOEt and D-AP5 ( $p<0.01$; paired Student's $t$ test). $\boldsymbol{H}$, Summary of the effects of CPCCOEt on SSE in PCs. norm., Normalized.

(Bisogno et al., 2003; Melis et al., 2004) greatly reduced the extent of retrograde inhibition for both PCs and SCs. Together, our results establish that SSE in SCs requires postsynaptic calcium increases and DAG lipase activation and is mediated by the release of 2-AG.

Thus far, our results suggest that endocannabinoids released from SCs inhibit transmitter release by acting on presynaptic CB1Rs; however, there is evidence for expression of other types of cannabinoid receptors (Breivogel et al., 2001; Hajos et al., 2001), some of which have not been characterized on a molecular level. To test the involvement of CB1Rs in mediating retrograde inhibition in SCs, we performed experiments in mice lacking CB1Rs (Fig. 3D) (Zimmer et al., 1999). Here, conditioning trains reliably led to post-tetanic potentiation, whereas SSE was observed in wild-type mice. These results confirm that retrograde inhibition at SCs is indeed mediated by presynaptic CB1Rs.
Properties of endocannabinoid release and retrograde inhibition from SCs

The observation that SSE in SCs is sensitive to DAG lipase inhibitors suggests that endocannabinoid release from SCs is greatly reduced by inhibitors of mGluR1, as is the case for PCs; however, we found that although SCs express mGluR1 (Hamori et al., 1996; Karakossian and Otis, 2004), bath application of the mGluR1 inhibitor CPCCOEt $(100 \mu \mathrm{M})$ had only a small effect on SSE (Fig. 4A). In these experiments, CPCCOEt was bath applied after stable SSE was obtained. CPCCOEt did not significantly alter the amplitude of the initial EPSC or the extent of SSE. This is apparent in the summary of five experiments (Fig. $4 A$, left), in the average synaptic currents from a representative experiment for control conditions, and in the presence of CPCCOEt (Fig. $4 A$, right), and in the summary of the time course of retrograde inhibition (Fig. 4B).

In light of the fact that NMDA receptors (NMDARs) are expressed on SCs but not PCs, we considered the possibility that activation of these receptors in SCs might mediate SSE. We found that application of the NMDAR antagonist CPP (5 $\mu \mathrm{M})$ alone had no effect on retrograde inhibition (Fig. 4C,D); however, coapplication of CPCCOEt and CPP prevented retrograde inhibition (Fig. 4E,F) without affecting the amplitude of the initial EPSC.

Figure $4 G$ compares the pharmacological sensitivity of SSE between PCs and SCs. Although the magnitude of SSE in control conditions was similar for both cell types, CPCCOEt had a major effect on retrograde inhibition in PCs (Fig. $4 H$ ) but only a minor effect in SCs. Blocking both NMDARs and mGluR1s reduced retrograde inhibition at synapses onto SCs to a similar extent as blocking mGluR1 alone at synapses onto PCs. This indicates that, in contrast to PCs, SCs can release endocannabinoids in the absence of mGluR1 activation through an NMDAR-dependent mechanism.

\section{SSE in SCs requires activation of numerous PF inputs}

In SCs, both mGluR1s and NMDARs are expressed extrasynaptically (Carter and Regehr, 2000; Clark and Cull-Candy, 2002; Karakossian and Otis, 2004), suggesting that the activation of numerous PF inputs resulting in glutamate pooling and spillover from the synaptic cleft is required for SSE. To test this hypothesis, we activated PF inputs with brief bursts as described above, but at lower stimulus intensities to recruit a smaller number of PF inputs. SSE was not detected under these conditions (Fig. 5A); instead, post-tetanic potentiation with a magnitude similar $(1.77 \pm$ $0.15 ; n=7)$ to that produced by PF stimulation at higher intensities in the presence of AM251 (1.89 \pm 0.24$)$ (Fig. 3B) was observed (Fig. $5 B$ ). This establishes that robust retrograde inhibi- 

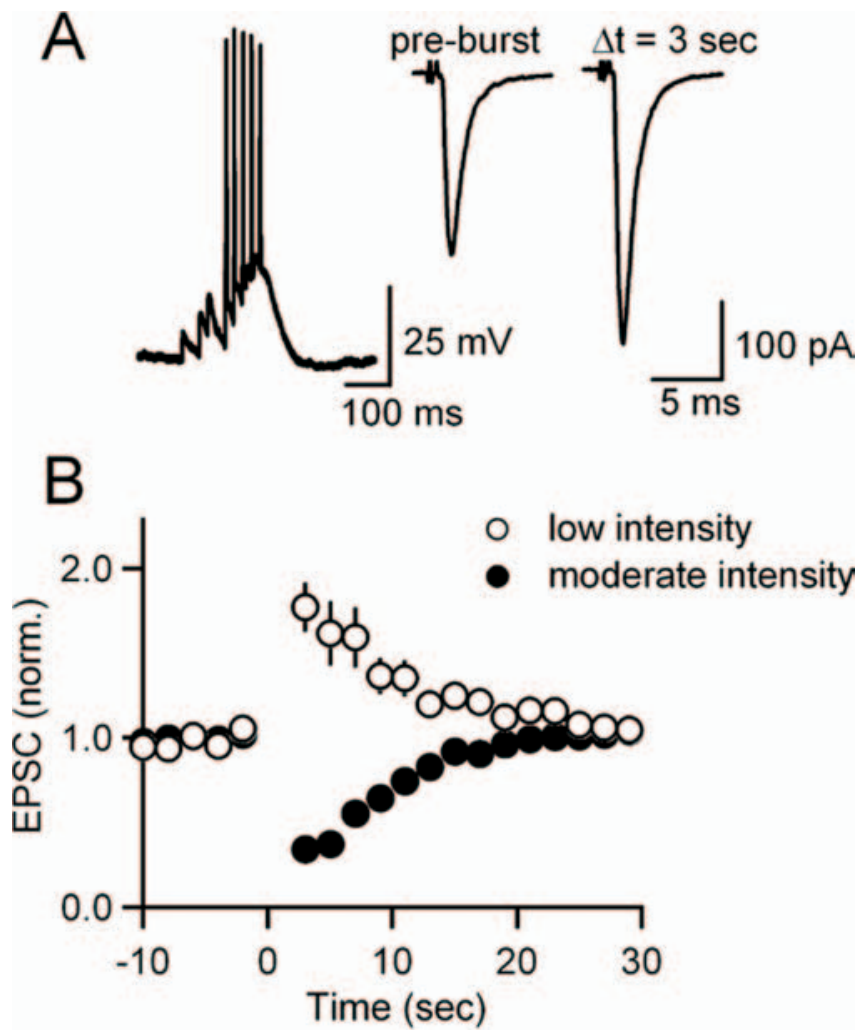

Figure 5. Low-intensity PF stimulation does not evoke SSE. A, Representative experiment, with the average response evoked by the conditioning train (left) and the average synaptic response recorded before and after the conditioning train (right). $\boldsymbol{B}$, Time course of synaptic enhancement after low-intensity stimulation (open circles). Time course of SSE after moderate intensity stimulation (closed circles; data are from Fig. 3B) is shown for comparison. norm., Normalized.

tion from SCs requires synchronous activation of numerous inputs and implies that glutamate pooling and spillover are necessary for SSE in SCs, similar to what has been observed in PCs (Brown et al., 2003; Marcaggi and Attwell, 2005). Moderate intensity stimulation was used for all of the following experiments.

\section{Endocannabinoids released from SCs do not mediate self-inhibition}

Previous studies have shown that SCs express CB1Rs on their postsynaptic membrane and that activation of these receptors, either by exogenous agonist application or by endocannabinoid release from nearby PCs, can lead to membrane hyperpolarization and a reduction of spontaneous firing (Kreitzer et al., 2002). Therefore, endocannabinoids released from SCs themselves could mediate autoinhibition.

We first tested whether endocannabinoid release evoked by PF bursts sufficient to activate presynaptic CB1Rs can also reach postsynaptic CB1Rs. SCs are spontaneously active, and their rate of firing is highly sensitive to CB1R activation (Kreitzer et al., 2002). We therefore examined the effect of PF bursts on spontaneous SC firing (Fig. 6A-C), which ranged from 5 to $15 \mathrm{~Hz}$; however, aside from a brief pause in firing immediately after the conditioning burst, SC firing frequency remained unaffected by PF bursts.

Recent studies have shown that certain types of neocortical interneurons can regulate their own excitability via an endocannabinoid-mediated mechanism. After several trains of action potentials, low-threshold spiking (LTS) interneurons show a persistent hyperpolarization of their membrane potential that is blocked by intracellular BAPTA or AM251 in the bath (Bacci et al., 2004). To test whether sustained high-frequency firing in SCs can mediate a similar form of self-inhibition, we evoked 10 trains of action potentials with depolarizing steps of $1 \mathrm{~s}$ duration, triggered every $20 \mathrm{~s}$ (Fig. 6D). Firing frequencies during the trains ranged from 20 to $40 \mathrm{~Hz}$ (Fig. $6 \mathrm{E}$ ); however, in contrast to cortical LTS neurons, SCs did not show a long-lasting change in their membrane potential (Fig. 6F).

Together, these results suggest that endocannabinoid release from SCs does not lead to self-inhibition, despite the expression of functional postsynaptic CB1Rs.

\section{Testing the functional consequences of endocannabinoid} release from interneurons

To further examine under what conditions endocannabinoids can be released from SCs and to test potential functional consequences, we performed a series of experiments in which SCs were held in current clamp. On the basis of our findings as well as previous results, there are several scenarios under which endocannabinoids could be released and exert their effect. Our observation that synaptic activation evokes endocannabinoid release from SCs and BCs implies that brief bursts could influence the ability of granule cells to trigger spikes in SCs. We also demonstrated that calcium increases in SCs and BCs as a result of depolarizing steps evoke widespread endocannabinoid release during DSE. It is possible that under more physiological conditions, spiking activity in SCs can also trigger widespread calcium increases and endocannabinoid release, resulting in a global decrease in synaptic efficacy. Thus endocannabinoids could either reduce synaptic efficacy by a pathway-specific release of endocannabinoids or by global release of endocannabinoids after global calcium increases.

We performed experiments to examine these possibilities (Fig. 7). We first recorded from SCs in current clamp, activated $\mathrm{PF}$ inputs at low frequency, and then tested the effect of PF activation with a brief burst (10 stimuli; $50 \mathrm{~Hz})($ Fig. $7 A-F)$. In these experiments, small hyperpolarizing currents (20-30 pA) were applied to suppress spontaneous activity, and stimulus intensities were adjusted to reliably $(p \sim 1)$ trigger a spike with a single stimulus (Fig. $7 B$, left). After stimulation with a burst (Fig. $7 A$ ), $\mathrm{PF}$ activation no longer triggered a spike in the SC (Fig. $7 B$, middle), but that ability recovered after $\sim 20 \mathrm{~s}$ (Fig. $7 B$, right). Bath application of AM251 did not affect the SC response to the PF burst (Fig. 7C), but bursts no longer affected the ability of PFs to trigger a spike (Fig. 7D). SC firing in this experiment is summarized in a raster plot (Fig. $7 E$ ). Similar results were obtained in four additional neurons (Fig. $7 F$ ). These findings establish that endocannabinoid release from SCs can transiently reduce the ability of PF synapses to trigger spikes in SCs.

It is possible that the spikes evoked during the conditioning burst contributed to the reduced ability of PF synapses to trigger an SC spike. We therefore evoked spikes with postsynaptic depolarizing steps of $300 \mathrm{~ms}$ duration instead of PF burst firing and monitored the ability of single stimuli to evoke spikes at low frequencies, before and after the depolarization (Fig. 7G-I). Under those conditions, high-frequency firing had no effect on the ability of PF synapses to evoke single spikes in SCs (Fig. $7 \mathrm{H}, I$ ). Thus, although depolarization produced more spikes than did PF bursts, no decrease in synaptic efficacy was apparent, indicating that high-frequency firing alone does not lead to widespread endocannabinoid release. Similarly, longer-lasting or multiple 
A

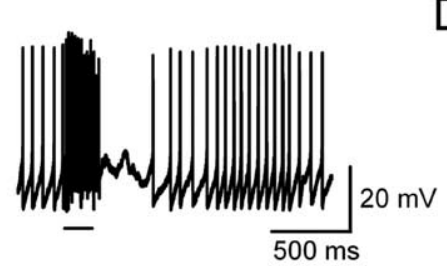

B
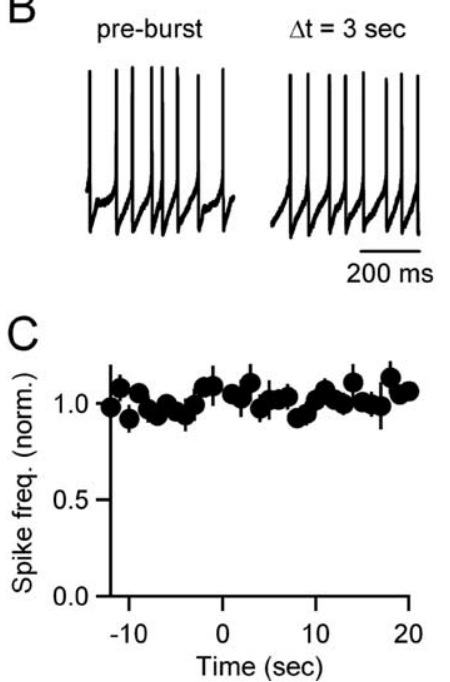

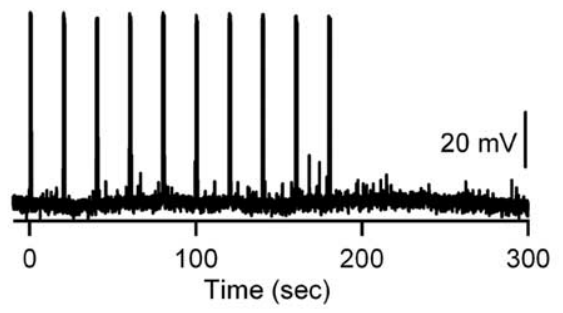

$E$
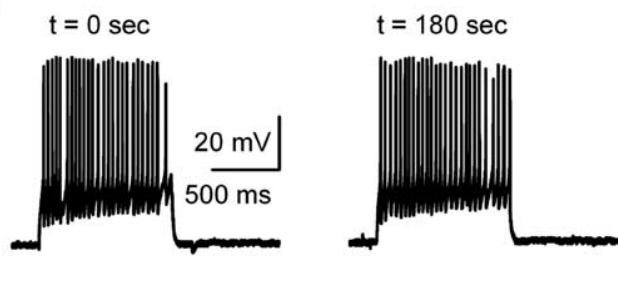

$\mathrm{F}$

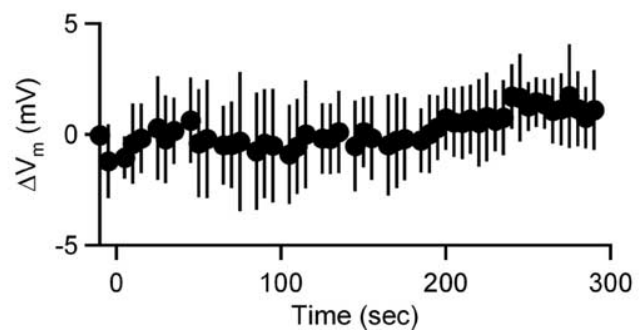

Figure 6. Endocannabinoids do not mediate autoinhibition in SCS. A-C, SCs were allowed to fire spontaneously, and the effect of synaptic activation (10 stimuli at $50 \mathrm{~Hz}$ ) on firing frequency was assessed. $\boldsymbol{A}$, Representative experiment showing PF bursts evoked during spontaneous activity. The bar underneath the trace indicates time of stimulation. Pause in firing after the burst was not influenced by AM251 application ( $n=3$ cells; data not shown). $\boldsymbol{B}$, Spontaneous firing before (left) and $3 \mathrm{~s}$ after (right) conditioning burst. $\boldsymbol{C}$, Normalized spike frequency (bin size $=1 \mathrm{~s}$ ) before and after conditioning bursts evoked at $t=0 \mathrm{~s} ; n=5$ cells. Frequency of firing ranged from 5 to 15 spikes/s. D-F, In a second set of experiments, SCs were hyperpolarized (20-30 pA) to suppress spontaneous firing, and the effects of spike firing evoked by current steps was assessed. $\boldsymbol{D}$, High-frequency trains of action potentials $(20-40 \mathrm{~Hz})$ evoked with $40-60 \mathrm{pA}$ depolarizing pulses of $1 \mathrm{~s}$ duration repeated 10 times did not lead to changes in resting membrane potential $\left(V_{m}\right)$ in a representative $S C$. $E$, Responses evoked by the 1st and 10th current step in cell shown in D. F, Summary of resting membrane potential after a series of current steps as in $\boldsymbol{D}$ ( $n=6$ cells). Spike freq., Spike frequency; norm., normalized.

trains of spikes had no effect on PF-evoked spike probability (data not shown).

These experiments establish that brief PF bursts lead to a local release of endocannabinoids that reduces the ability of PFs to trigger spikes in SCs via retrograde synaptic inhibition. As a consequence, feedforward inhibition mediated by SCs onto their postsynaptic targets should be transiently reduced after PF bursts. We tested this possibility by recording from PCs, the main targets of feedforward inhibition (Fig. 7J) of molecular layer interneurons (Eccles et al., 1967; Mittmann et al., 2005). PCs were voltage clamped at the reversal potential of excitatory inputs $(0$ $\mathrm{mV}$ ), and endocannabinoid release from PCs was blocked by the inclusion of BAPTA $(20 \mathrm{~mm})$ in the recording pipette. PF inputs were activated at large lateral distances $(<100 \mu \mathrm{m})$ to limit the activation of monosynaptic inhibitory inputs. Disynaptic IPSCs were evoked at short latencies (Fig. $7 K$ ). After PF bursts (10 stimuli; $50 \mathrm{~Hz}$ ), IPSC amplitude was transiently reduced to $0.56 \pm$ 0.06 of control ( $n=7$ cells). Bath application of AM251 attenuated this reduction in all cells to $0.79 \pm 0.07$ of control (Fig. $7 K, L$ ).

Together, our results show that SSE at PF to SC synapses can transiently reduce synapse-evoked firing in SCs, thereby leading to a reduction in feedforward inhibition in PCs.

\section{Discussion}

Here we have shown that local interneurons in the cerebellum release endocannabinoids that transiently inhibit PF synaptic inputs. We found that SSE is mediated by the activation of either mGluR1s or NMDARs. Moreover, the activation of multiple PF inputs was required to induce SSE. This is consistent with the extrasynaptic location of both mGluRs and NMDARs, which requires the activation of multiple closely spaced synapses that results in the pooling of glutamate and spillover from the synaptic cleft. SSE led to a transient reduction of the ability of PF inputs to trigger spikes in the postsynaptic SC. As a consequence, feedforward inhibition exerted by SCs and BCs onto PCs and other postsynaptic targets was dynamically regulated.

\section{Mechanisms of endocannabinoid release from stellate cells}

SCs release endocannabinoids in response to either postsynaptic depolarization or brief bursts of high-frequency synaptic activation. Calcium increases and DAG lipase activation in postsynaptic SCs are crucial in mediating SSE at PF synapses. This finding contrasts with previous results at the same cell type, where a transient suppression of release probability after brief bursts was attributed to endocannabinoid spillover from neighboring PCs (Rancillac and Barbara, 2005). The involvement of DAG lipase suggests that $2-A G$ is the endocannabinoid released from SCs in response to synaptic activation. Despite similarities to endocannabinoid release from PCs, release from SCs is distinct in important ways. For PF to PC synapses, mGluR1 antagonists greatly reduce synaptically evoked retrograde inhibition (Brown et al., 2003; Brenowitz and Regehr, 2005), indicating that glutamate spillover and pooling lead to the activation of extrasynaptic mGluR1s on PCs. In contrast, mGluR1 antagonists alone barely alter retrograde inhibition at PF to SC synapses, although SCs also express mGluR1 (Hamori et al., 1996; Karakossian and Otis, 2004). Although application of an NMDAR antagonist had only a minor effect on retrograde inhibition, blocking both NMDARs and mGluR1s greatly attenuated retrograde inhibition at PF to SC synapses. This suggests that, although mGluR1 contributes to 2-AG release from both cell types, mGluR1 activation is essential in PCs, whereas activation of either NMDARs or mGluR1s is sufficient to evoke the release of 2-AG from SCs.

These findings indicate a novel role for NMDARs in the release of endocannabinoids from SCs. Previous studies at the PF to SC synapse have shown that the NMDAR component of the PF EPSC is apparent only for high-intensity stimulation or after stimulus trains (Carter and Regehr, 2000; Clark and Cull-Candy, 2002). This suggests that glutamate pooling and spillover are important in the activation of extrasynaptic NMDARs. Based on the 
similar high affinity and extrasynaptic location of mGluR1s and NMDARs, it appears that NMDARs and mGluR1s play similar roles in SCs. They both have a high affinity for glutamate and are located at extrasynaptic sites that are suited for detecting glutamate that has escaped from the synaptic cleft.

\section{Endocannabinoid release \\ from interneurons}

Our results are the first demonstration of retrograde inhibition of synaptic inputs mediated by endocannabinoid release from interneurons. Previous studies of inhibitory interneurons did not observe endocannabinoid-mediated effects on synaptic strength (Hoffman et al., 2003; Patenaude et al., 2005), suggesting that the synthesis and release of endocannabinoids is not a general property of neurons. In the only previous description of endocannabinoid release from interneurons (Bacci et al., 2004), sustained firing from a specific type of cortical interneuron led to a longlasting endocannabinoid-mediated autocrine persistent decrease in excitability (termed slow self-inhibition) mediated by postsynaptic $\mathrm{CB} 1$ receptors (retrograde synaptic inhibition was not examined). In contrast, although SCs and BCs express postsynaptic CB1Rs whose activation can suppress firing, neither spike trains nor $\mathrm{PF}$ bursts in SCs led to their activation. Selfinhibition was not apparent even when retrograde synaptic inhibition was prominent. Thus, cerebellar interneurons use endocannabinoids primarily to retrogradely control synaptic inputs rather than to regulate their own firing.

\section{Functional consequences of SSE by interneurons}

Interneurons throughout the brain are part of feed-forward inhibitory circuits that tightly control the spike timing of principal neurons (Carter and Regehr, 2002; Pouille and Scanziani, 2004; Blitz and Regehr, 2005; Gabernet et al., 2005; Mittmann et al., 2005). For the cerebellum, most theories on the control of movement assume that the timing of action potentials in PCs in response to sensory activation is precisely controlled by the cerebellar cortical circuitry. In large part, this may be achieved by feed-forward inhibition of PCs mediated by both BCs and SCs (Eccles et al., 1967). Anatomical and functional studies suggest that PCs are directly excited and then rapidly inhibited by interneurons that respond to the same set of active PF inputs (Eccles et al., 1967; Sultan and Bower, 1998; Brunel et al., 2004; Mittmann et al., 2005). Thus, this circuitry might provide a narrow time window in which $\mathrm{PF}$ inputs onto PCs can be integrated effectively.
A

B

E
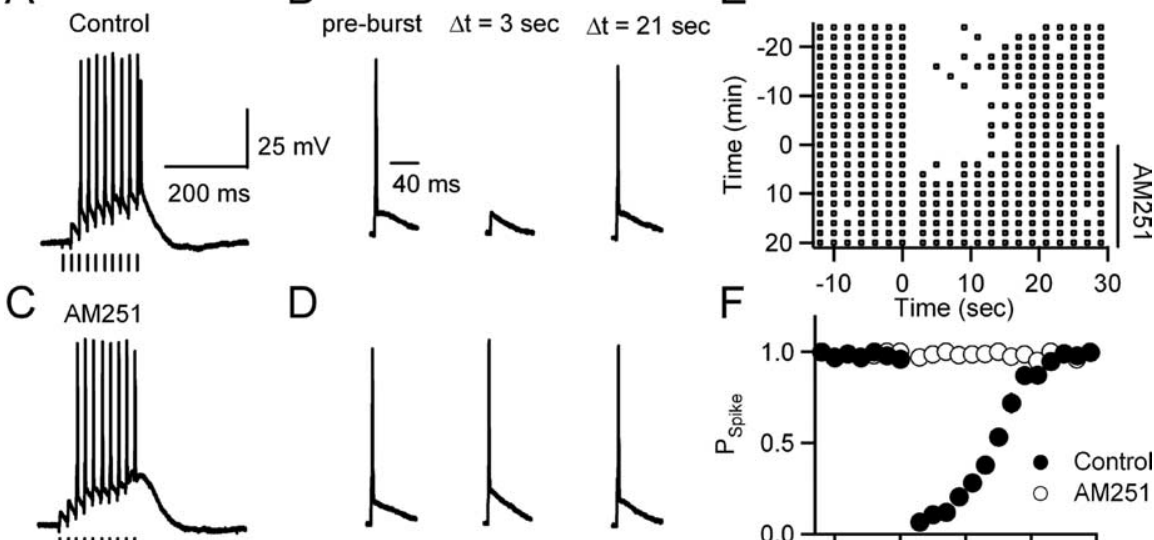

Postsynaptic

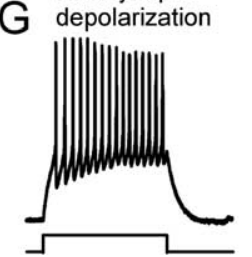

H
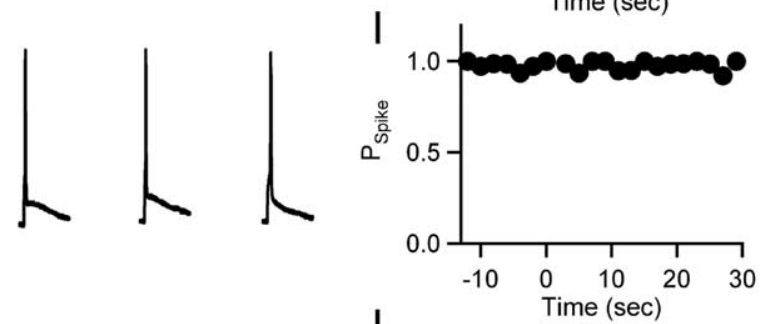

$J$

$\mathrm{K}$

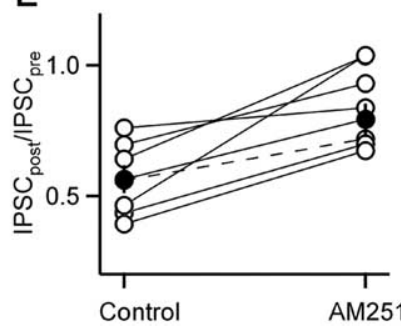

Figure 7. Control of SC spiking and disynaptic inhibition by SSE. $\boldsymbol{A}-\boldsymbol{F}, \mathrm{PF}$ synapses were activated at $0.5 \mathrm{~Hz}$ before and after a brief PF burst ( 10 stimuli; $50 \mathrm{~Hz}$ ) at $t=0 \mathrm{~s}$. Trials were repeated every $2 \mathrm{~min}$, and $2 \mu \mathrm{M}$ AM251 was bath applied. The resting potential was maintained at $-70 \mathrm{mV}$ to suppress spontaneous firing. Stimulus durations for conditioning bursts were lower (150 $\mu \mathrm{s})$ than for low-frequency stimuli (200 $\mu \mathrm{S})$. $A, C$, Typical $S C$ responses are shown for a representative experiment evoked by the burst in control conditions $(\boldsymbol{A})$ and in the presence of $\operatorname{AM} 251(\boldsymbol{C}) . \boldsymbol{B}, \boldsymbol{D}$, The response to a single PF activation is shown before the burst and 3 and $21 \mathrm{~s}$ after the burst in control conditions $(\boldsymbol{B})$ and in the presence of AM251 (D). $\boldsymbol{E}$, The SC firing for this experiment is shown in a raster plot, with AM251 applied in the bath at time $t=0 \mathrm{~min}$ (vertical axis). Squares indicate individual action potentials. Conditioning bursts were applied at time $t=0 \mathrm{~s}$ (horizontal axis). $\boldsymbol{F}$, The probability of PF inputs evoking a spike in an $\mathbf{S C}$ as in $\boldsymbol{A}-\boldsymbol{E}$, before and after a conditioning burst applied at $t=0(n=5)$. $\mathbf{G}-\boldsymbol{I}$, Experiments as in $\boldsymbol{A}-\boldsymbol{F}$, but at $t=0 \mathrm{~S}$, postsynaptic spike trains were evoked with depolarizing steps $(300 \mathrm{~ms} ; 20-30 \mathrm{pA})$ rather than with PF bursts. $\boldsymbol{G}$, The responses to depolarization $(\boldsymbol{H})$ and to PF stimulation at different time points are shown for a representative experiment. $\boldsymbol{I}$, The probability of PF activation evoking a spike in an SC as in $\boldsymbol{G}$ and $\boldsymbol{H} ; \boldsymbol{n}=5$. J-L, SSE controls feedforward inhibition. J, Schematic of feedforward inhibitory circuitry. $\boldsymbol{K}$, Disynaptic IPSCs recorded in PCs in voltage clamp, at a holding potential of $0 \mathrm{mV}$. Responses were evoked before (IPSC $\mathrm{pre}_{\text {pre }}$ ) and $5 \mathrm{~s}$ after (IPSC $\mathrm{post}_{\text {s }}$ ) brief burst of $10 \mathrm{PF}$ stimuli $(50 \mathrm{~Hz})$, under control conditions (black traces) and after application of AM251 (gray traces). L, Summary of IPSC post $_{\text {IPSC }}$ pre ratios in control conditions and in the presence of AM251 (open circles; $n=7$ cells). Closed circles indicate average, and dashed line indicates cell shown in $\boldsymbol{K} . P_{\text {spike, }}$ Spike probability.

Here we have shown that brief bursts can transiently reduce the ability of PF inputs to generate action potentials in SCs because of SSE. Thus, high-frequency bursts in granule cells can transiently reduce feedforward inhibition in PCs. This might allow PCs to effectively integrate incoming excitatory inputs over a longer time window, thereby promoting certain forms of shortor long-term plasticity.

\section{References}

Bacci A, Huguenard JR, Prince DA (2004) Long-lasting self-inhibition of neocortical interneurons mediated by endocannabinoids. Nature 431:312-316. 
Beierlein M, Regehr WG (2006) Brief bursts of parallel fiber activity trigger calcium signals in Bergmann glia. J Neurosci 26:6958-6967.

Bisogno T, Howell F, Williams G, Minassi A, Cascio MG, Ligresti A, Matias I, Schiano-Moriello A, Paul P, Williams EJ, Gangadharan U, Hobbs C, Di Marzo V, Doherty P (2003) Cloning of the first sn1-DAG lipases points to the spatial and temporal regulation of endocannabinoid signaling in the brain. J Cell Biol 163:463-468.

Bisogno T, Ligresti A, Di Marzo V (2005) The endocannabinoid signaling system: biochemical aspects. Pharmacol Biochem Behav 81:224-238.

Blitz DM, Regehr WG (2005) Timing and specificity of feed-forward inhibition within the LGN. Neuron 45:917-928.

Breivogel CS, Griffin G, Di Marzo V, Martin BR (2001) Evidence for a new $G$ protein-coupled cannabinoid receptor in mouse brain. Mol Pharmacol 60:155-163.

Brenowitz SD, Regehr WG (2005) Associative short-term synaptic plasticity mediated by endocannabinoids. Neuron 45:419-431.

Brown SP, Brenowitz SD, Regehr WG (2003) Brief presynaptic bursts evoke synapse-specific retrograde inhibition mediated by endogenous cannabinoids. Nat Neurosci 6:1048-1057.

Brown SP, Safo PK, Regehr WG (2004) Endocannabinoids inhibit transmission at granule cell to Purkinje cell synapses by modulating three types of presynaptic calcium channels. J Neurosci 24:5623-5631.

Brunel N, Hakim V, Isope P, Nadal JP, Barbour B (2004) Optimal information storage and the distribution of synaptic weights: perceptron versus Purkinje cell. Neuron 43:745-757.

Carter AG, Regehr WG (2000) Prolonged synaptic currents and glutamate spillover at the parallel fiber to stellate cell synapse. J Neurosci 20:4423-4434.

Carter AG, Regehr WG (2002) Quantal events shape cerebellar interneuron firing. Nat Neurosci 5:1309-1318.

Chadderton P, Margrie TW, Hausser M (2004) Integration of quanta in cerebellar granule cells during sensory processing. Nature 428:856-860.

Chevaleyre V, Takahashi KA, Castillo PE (2006) Endocannabinoidmediated synaptic plasticity in the CNS. Annu Rev Neurosci 29:37-76.

Clark BA, Cull-Candy SG (2002) Activity-dependent recruitment of extrasynaptic NMDA receptor activation at an AMPA receptor-only synapse. J Neurosci 22:4428-4436.

Diana MA, Marty A (2004) Endocannabinoid-mediated short-term synaptic plasticity: depolarization-induced suppression of inhibition (DSI) and depolarization-induced suppression of excitation (DSE). Br J Pharmacol 142:9-19.

Dittman JS, Regehr WG (1997) Mechanism and kinetics of heterosynaptic depression at a cerebellar synapse. J Neurosci 17:9048-9059.

Eccles JC, Ito M, Szentagothai J (1967) The cerebellum as a neuronal machine. New York: Springer.

Egertova M, Elphick MR (2000) Localisation of cannabinoid receptors in the rat brain using antibodies to the intracellular C-terminal tail of $\mathrm{CB}$. J Comp Neurol 422:159-171.

Gabernet L, Jadhav SP, Feldman DE, Carandini M, Scanziani M (2005) Somatosensory integration controlled by dynamic thalamocortical feedforward inhibition. Neuron 48:315-327.

Hajos N, Ledent C, Freund TF (2001) Novel cannabinoid-sensitive receptor mediates inhibition of glutamatergic synaptic transmission in the hippocampus. Neuroscience 106:1-4.

Hamori J, Takacs J, Gorcs TJ (1996) Immunocytochemical localization of mGluRla metabotropic glutamate receptor in inhibitory interneurons of the cerebellar cortex. Acta Biol Hung 47:181-194.

Hashimotodani Y, Ohno-Shosaku T, Tsubokawa H, Ogata H, Emoto K, Maejima T, Araishi K, Shin HS, Kano M (2005) Phospholipase Cbeta serves as a coincidence detector through its $\mathrm{Ca}^{2+}$ dependency for triggering retrograde endocannabinoid signal. Neuron 45:257-268.

Hoffman AF, Riegel AC, Lupica CR (2003) Functional localization of cannabinoid receptors and endogenous cannabinoid production in distinct neuron populations of the hippocampus. Eur J Neurosci 18:524-534.

Karakossian MH, Otis TS (2004) Excitation of cerebellar interneurons by group I metabotropic glutamate receptors. J Neurophysiol 92:1558-1565.

Kreitzer AC, Regehr WG (2001a) Retrograde inhibition of presynaptic calcium influx by endogenous cannabinoids at excitatory synapses onto Purkinje cells. Neuron 29:717-727.

Kreitzer AC, Regehr WG (2001b) Cerebellar depolarization-induced suppression of inhibition is mediated by endogenous cannabinoids. J Neurosci 21:RC174(1-5).

Kreitzer AC, Regehr WG (2002) Retrograde signaling by endocannabinoids. Curr Opin Neurobiol 12:324-330.

Kreitzer AC, Carter AG, Regehr WG (2002) Inhibition of interneuron firing extends the spread of endocannabinoid signaling in the cerebellum. Neuron 34:787-796.

Maejima T, Hashimoto K, Yoshida T, Aiba A, Kano M (2001) Presynaptic inhibition caused by retrograde signal from metabotropic glutamate to cannabinoid receptors. Neuron 31:463-475.

Maejima T, Oka S, Hashimotodani Y, Ohno-Shosaku T, Aiba A, Wu D, Waku K, Sugiura T, Kano M (2005) Synaptically driven endocannabinoid release requires $\mathrm{Ca}^{2+}$-assisted metabotropic glutamate receptor subtype 1 to phospholipase $\mathrm{C} \beta 4$ signaling cascade in the cerebellum. J Neurosci 25:6826-6835.

Marcaggi P, Attwell D (2005) Endocannabinoid signaling depends on the spatial pattern of synapse activation. Nat Neurosci 8:776-781.

Melis M, Perra S, Muntoni AL, Pillolla G, Lutz B, Marsicano G, Di Marzo V, Gessa GL, Pistis M (2004) Prefrontal cortex stimulation induces 2-arachidonoyl-glycerol-mediated suppression of excitation in dopamine neurons. J Neurosci 24:10707-10715.

Mittmann W, Koch U, Hausser M (2005) Feed-forward inhibition shapes the spike output of cerebellar Purkinje cells. J Physiol (Lond) 563:369-378.

Ohno-Shosaku T, Maejima T, Kano M (2001) Endogenous cannabinoids mediate retrograde signals from depolarized postsynaptic neurons to presynaptic terminals. Neuron 29:729-738.

Palay SL, Chan-Palay V (1974) Cerebellar Cortex. New York: Springer.

Patenaude C, Massicotte G, Lacaille JC (2005) Cell-type specific GABA synaptic transmission and activity-dependent plasticity in rat hippocampal stratum radiatum interneurons. Eur J Neurosci 22:179-188.

Piomelli D (2003) The molecular logic of endocannabinoid signaling. Nat Rev Neurosci 4:873-884.

Pouille F, Scanziani M (2004) Routing of spike series by dynamic circuits in the hippocampus. Nature 429:717-723.

Rancillac A, Barbara JG (2005) Frequency-dependent recruitment of inhibition mediated by stellate cells in the rat cerebellar cortex. J Neurosci Res 80:414-423.

Sultan F, Bower JM (1998) Quantitative Golgi study of the rat cerebellar molecular layer interneurons using principal component analysis. J Comp Neurol 393:353-373.

Wilson RI, Nicoll RA (2001) Endogenous cannabinoids mediate retrograde signaling at hippocampal synapses. Nature 410:588-592.

Zimmer A, Zimmer AM, Hohmann AG, Herkenham M, Bonner TI (1999) Increased mortality, hypoactivity, and hypoalgesia in cannabinoid CB1 receptor knockout mice. Proc Natl Acad Sci USA 96:5780-5785.

Zucker RS, Regehr WG (2002) Short-term synaptic plasticity. Annu Rev Physiol 64:355-405. 